\title{
Cone asymptotes of convex sets
}

\author{
V. Soltan \\ Department of Mathematical Sciences, George Mason University \\ 4400 University Drive, Fairfax, VA 22030, USA \\ vsoltan@gmu.edu
}

Received November 18, 2020

Accepted November 28, 2020

Presented by Horst Martini

Abstract: Based on the notion of plane asymptote, we introduce the new concept of cone asymptote of a set in the $n$-dimensional Euclidean space. We discuss the existence and describe some families of cone asymptotes.

Key words: Plane asymptote, cone asymptote, convex set.

MSC (2020): 52A20, 90C25.

\section{INTRODUCTION}

Originated in geometry, a widely used definition of an asymptote says that it is a line (or a halfline) that continually approaches a curve or a surface but does not meet it. In convex geometry, this definition was generalized by Gale and Klee [2], who defined an asymptote of a nonempty closed subset $X$ of the Euclidean space $\mathbb{R}^{n}$ as a halfline $h$ which lies in $\mathbb{R}^{n} \backslash X$ and satisfies the condition $\delta(X, h)=0$, where the inf-distance $\delta(X, Y)$ between nonempty sets $X$ and $Y$ in $\mathbb{R}^{n}$ is given by

$$
\delta(X, Y)=\inf \{\|x-y\|: x \in X, y \in Y\} .
$$

Later, Klee [4 introduced the concept of $j$-asymptote of a closed convex set $K \subset \mathbb{R}^{n}$, as a plane $L \subset \mathbb{R}^{n}$ of dimension $j, 1 \leq j \leq n-1$, satisfying the conditions $K \cap L=\varnothing$ and $\delta(K, L)=0$. In particular, a 1-asymptote is a line asymptote (see Figure 1).

Plane asymptotes appeared to be a useful tool in the study of various properties of convex sets. For instance, closed convex sets in $\mathbb{R}^{n}$ without plane asymptotes are precisely those whose affine images are closed (see [1, 5, 9]). Certain classes of convex sets (like continuous convex sets, M-decomposable sets, and M-polyhedral sets) have suitable geometric properties due to the

ISSN: 0213-8743 (print), 2605-5686 (online)

(C) The author(s) - Released under a Creative Commons Attribution License (CC BY-NC 3.0) 


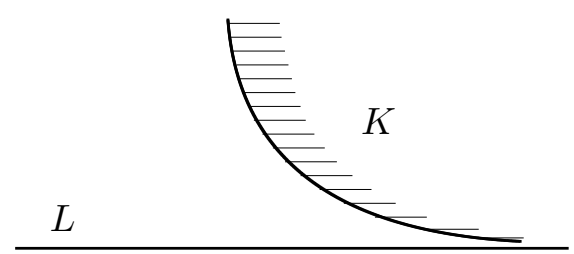

Figure 1: A plane asymptote $L$ of a convex set $K$.

absence of plane asymptotes (see [2, 4, 17, 8, 11]). The range of dimensions of plane asymptotes of a given closed convex set in $\mathbb{R}^{n}$ was investigated in [3, 5].

In the study of asymptotic behavior of convex sets, it is often desirable to describe their line or halfline asymptotes. This task is often difficult (if possible at all) partly due to the fact that the family of plane asymptotes is not hereditary (see, for instance, Example 3.5 below). Our goal here is to show that plane asymptotes of a given convex set $K \subset \mathbb{R}^{n}$ always contain closed cones which are asymptotes of $K$ (see Theorem 3.3 and Theorem 4.1 below). Also, we study some geometric properties of cone asymptotes.

For terminological convenience, we will say that a nonempty closed set $X \subset \mathbb{R}^{n}$ is an asymptote of a nonempty closed set $Y \subset \mathbb{R}^{n}$ provided $X \cap Y=\varnothing$ and $\delta(X, Y)=0$. Furthermore, we use the expression cone asymptote to distinguish it from another established concept, asymptotic cone $C_{X}$ of a nonempty set $X \subset \mathbb{R}^{n}$, which is a cone with apex at the origin $o$, defined by $C_{X}=\bigcap(\operatorname{cl}((0, \varepsilon] X): \varepsilon>0)$.

We conclude this section with necessary definitions, notation, and results on convex sets in the $n$-dimensional Euclidean space $\mathbb{R}^{n}$ (see, e.g., [10] for details). The elements of $\mathbb{R}^{n}$ are called vectors, or points. In what follows, $o$ stands for the zero vector of $\mathbb{R}^{n}$. We denote by $[u, v]$, and $[u, v\rangle$, respectively, the closed segment with endpoints $u, v \in \mathbb{R}^{n}$ and the closed halfline through $v$ with endpoint $u$ :

$$
\begin{aligned}
& {[u, v]=\{(1-\lambda) u+\lambda v: 0 \leq \lambda \leq 1\}} \\
& {[u, v\rangle=\{(1-\lambda) u+\lambda v: \lambda \geq 0\}}
\end{aligned}
$$

Also, $u \cdot v$ will mean the dot product of $u$ and $v$, and $\|u\|$ denotes the norm of $u$.

Given a pair of subspaces $T \subset S$ of $\mathbb{R}^{n}$, a subspace $F \subset \mathbb{R}^{n}$ will be called complementary to $T$ in $S$ provided $F \cap T=\{o\}$ and $F+T=S$. By an $r$-dimensional plane $L$ in $\mathbb{R}^{n}$, where $0 \leq r \leq n$, we mean a translate of 
a suitable $r$-dimensional subspace $S_{L}$ of $\mathbb{R}^{n}: L=u+S_{L}$, where $u \in \mathbb{R}^{n}$. The subspace $S_{L}$ is uniquely determined by $L$ and equals $L-v$ for any choice of $v \in L$.

A closed halfspace $V$ of $\mathbb{R}^{n}$ is defined by $V=\left\{x \in \mathbb{R}^{n}: x \cdot e \leq \gamma\right\}$, where $e$ is a nonzero vector and $\gamma$ is a scalar. If $L \subset \mathbb{R}^{n}$ is a plane of positive dimension, then by a closed halfplane of $L$ we will mean any set of the form $L \cap V$, where $V$ is a closed halfspace of $\mathbb{R}^{n}$ satisfying the condition $\varnothing \neq L \cap V \neq L$.

In what follows, $K$ means a nonempty convex set in $\mathbb{R}^{n}$. The relative interior and relative boundary of $K$ are denoted $\operatorname{rint} K$ and $\operatorname{rbd} K$, respectively. A convex set $K \subset \mathbb{R}^{n}$ is called line-free if it contains no lines. For a nonempty set $X \subset \mathbb{R}^{n}$, the notations cl $X$ and conv $X$ stand, respectively, for the closure and the convex hull of $X$. If $X$ is bounded, then the set $Y=\operatorname{conv}(\operatorname{cl} X)$ is compact.

We recall that a nonempty set $C$ in $\mathbb{R}^{n}$ is a cone with apex $v \in \mathbb{R}^{n}$ if $v+\lambda(x-v) \in C$ whenever $\lambda \geq 0$ and $x \in C$. (Obviously, this definition implies that $v \in C$, although a stronger condition $\lambda>0$ can be beneficial; see, e.g., [6.) A cone $C$ with apex $v$ is called convex if it is a convex set, and is called nontrivial if $C \neq\{v\}$. The polar cone $C^{\circ}$ of a convex cone $C \subset \mathbb{R}^{n}$ with apex $o$ is defined by

$$
C^{\circ}=\left\{x \in \mathbb{R}^{n}: x \cdot e \leq 0 \quad \forall e \in C\right\} .
$$

It is known that $C^{\circ}$ is a closed convex cone with apex $o$. Furthermore, $C^{\circ}$ is $n$-dimensional provided $C$ is line-free.

For a convex set $K \subset \mathbb{R}^{n}$ and a point $v \in \mathbb{R}^{n}$, the generated cone $C_{v}(K)$ is defined by

$$
C_{v}(K)=\{v+\lambda(x-v): x \in K, \lambda \geq 0\} .
$$

Both sets $C_{v}(K)$ and $\operatorname{cl} C_{v}(K)$ are convex cones with apex $v$.

The recession cone of a convex set $K \subset \mathbb{R}^{n}$ is defined by

$$
\operatorname{rec} K=\left\{e \in \mathbb{R}^{n}: x+\lambda e \in K \text { whenever } x \in K \text { and } \lambda \geq 0\right\},
$$

and the lineality space of $K$ is the subspace defined by

$$
\operatorname{lin} K=\operatorname{rec} K \cap(-\operatorname{rec} K) .
$$

We will use the following properties of recession cones and lineality spaces of closed convex sets.

(P1) rec $K$ is a closed convex cone with apex $o$; it is distinct from $\{o\}$ if and only if $K$ is unbounded. 
(P2) For any given point $u \in K$, the cone rec $K$ is the largest among all closed convex cones $C \subset \mathbb{R}^{n}$ with apex $o$ satisfying the inclusion $u+C \subset K$.

(P3) One has $K+\operatorname{lin} K=K$.

(P4) If $C \subset \mathbb{R}^{n}$ is a closed convex cone with apex $o$, then $\operatorname{lin} C$ is the largest subspace contained in $C$.

\section{Halfplane asymptotes}

Klee 4 (also see [3]) observed that a line asymptote of a closed convex set $K \subset \mathbb{R}^{n}$ contains a halfline asymptote. Our first result partly generalizes this assertion to the case of halfplanes of a plane asymptote.

Theorem 2.1. If $K \subset \mathbb{R}^{n}$ is a closed convex set and $L \subset \mathbb{R}^{n}$ is a plane asymptote of $K$, then the following assertions hold.

(a) For any bounded set $X \subset L$, there is a closed halfplane of $L$, disjoint from $X$, which is an asymptote of $K$.

(b) For any bounded set $Z \subset L$, there is a closed halfplane of $L$, containing $Z$, which is an asymptote of $K$.

Proof. (a) Consider the sets

$$
M_{r}=\{x \in L: \delta(K, x) \leq 1 / r\}, \quad r \geq 1
$$

We state that every set $M_{r}$ is nonempty, closed, and convex. Indeed, since $\delta(K, L)=0$, there are points $p \in K$ and $q \in L$ such that $\|p-q\| \leq 1 / r$. Obviously, $\delta(K, q) \leq\|p-q\| \leq 1 / r$, which gives the inclusion $q \in M_{r}$. If a sequence of points $q_{1}, q_{2}, \ldots \in M_{r}$ converges to a point $q$, then $q \in L$ due to the closedness of $L$. Because the function $\delta(K, x)$ continuously depends on $x \in \mathbb{R}^{n}$ (see, e.g., [10, Theorem 8.21]), one has

$$
\delta(K, q)=\lim _{i \rightarrow \infty} \delta\left(K, q_{i}\right) \leq 1 / r
$$

implying the inclusion $q \in M_{r}$. So, the set $M_{r}$ is closed.

For the convexity of $M_{r}$, choose any points $q, q^{\prime} \in M_{r}$ and a scalar $\varepsilon>0$. There are points $p, p^{\prime} \in K$ such that

$$
\|p-q\| \leq \delta(K, q)+\varepsilon \leq 1 / r+\varepsilon, \quad\left\|p^{\prime}-q^{\prime}\right\| \leq \delta\left(K, q^{\prime}\right)+\varepsilon \leq 1 / r+\varepsilon .
$$


Given a scalar $\lambda \in[0,1]$, put

$$
p_{0}=(1-\lambda) p+\lambda p^{\prime} \quad \text { and } \quad q_{0}=(1-\lambda) q+\lambda q^{\prime} .
$$

Then $p_{0} \in K$ by the convexity of $K$, and $q_{0} \in L$ because $(1-\mu) q+\mu q^{\prime} \in L$ whenever $\mu \in \mathbb{R}$ (see [10, Theorem 2.38]). Furthermore,

$$
\begin{aligned}
\delta\left(K, q_{0}\right) & \leq\left\|p_{0}-q_{0}\right\| \\
& \leq(1-\lambda)\|p-q\|+\lambda\left\|p^{\prime}-q^{\prime}\right\| \leq 1 / r+\varepsilon \quad \text { for each } \varepsilon>0 .
\end{aligned}
$$

Thus $\delta\left(K, q_{0}\right) \leq 1 / r$, which gives $q_{0} \in M_{r}$. So, the set $M_{r}$ is convex.

Next, we observe that $M_{1} \cap M_{2} \cap \cdots=\varnothing$. Indeed, assuming the existence of a point $u \in M_{1} \cap M_{2} \cap \cdots \subset L$, we would have

$$
\delta(K, u) \leq \inf \{1 / r: r \geq 1\}=0,
$$

which gives $u \in K$. The latter contradicts the assumption $K \cap L=\varnothing$.

Let $X$ be a bounded subset of $L$. Then the convex set $Y=\operatorname{conv}(\operatorname{cl} X)$ is compact. By the above argument, $Y \cap M_{1} \cap M_{2} \cap \cdots=\varnothing$. Since the sequence of closed sets $M_{1}, M_{2}, \ldots$ is decreasing, the compactness of $Y$ implies the existence of an integer $t$ such that $Y \cap M_{r}=\varnothing$ for all $r \geq t$. Furthermore, $\delta\left(Y, M_{t}\right)>0$. Hence there is a hyperplane $H \subset \mathbb{R}^{n}$ separating $Y$ and $M_{t}$ such that these sets are contained in the opposite open halfspaces determined by $H$ (see, e.g., [10, Theorem 10.12]). Denote by $V$ the closed halfspace determined by $H$ and containing $M_{t}$, and let $P=L \cap V$. Clearly, $P$ is a closed halfplane of $L$ containing $M_{t}$ and disjoint from $Y$. Furthermore,

$$
\delta(K, P) \leq \inf \left\{\delta\left(K, M_{r}\right): r \geq t\right\} \leq \inf \{1 / r: r \geq t\}=0 .
$$

Summing up, $P$ is an asymptote of $K$.

(b) Let $Z$ be a bounded subset of $L$, and let $P$ be a closed halfplane of $L$ which is an asymptote of $K$ (the existence of $P$ is proved above). By a compactness argument, one can choose a translate $v+P$ of $P$ which lies in $L$ and contains $P \cup Z$. Obviously, the halfplane $v+P$ is an asymptote of $K$.

We observe that the method of proof of Theorem 2.1 gives a limited choice of halfplane asymptotes of $K$, as illustrated by the next example.

Example 2.2. Consider $K=\{(x, y, z): x>0, y \geq 1 / x\}$, closed convex set in $\mathbb{R}^{3}$. It is easy to see that the coordinate $x z$-plane of $\mathbb{R}^{3}$, say $L$, is an asymptote of $K$. The sets $M_{r} \subset L$ defined in Theorem 2.1 are vertical closed 
halfplanes of $L$ of the form $\left\{(x, 0, z): x \geq \alpha_{r}\right\}, r \geq 1$. Consequently, the halfplane asymptote $P$ of $K$ described in Theorem 2.1 should be of a similar form. On the other hand, it is easy to see that the only closed halfplanes of $L$, which are not asymptotes of $K$, have the form $\{(x, 0, z): x \leq \lambda\}$.

\section{Cone asymptotes}

We are going to refine the argument of Theorem 2.1 to describe a wider family of asymptotes which are subsets of a given plane asymptote. For a nontrivial closed convex cone $C \subset \mathbb{R}^{n}$ with apex $o$, consider its conic $\varepsilon$ neighborhood $D_{\varepsilon}(C), \varepsilon>0$, defined as the union of all closed halflines $h \subset \mathbb{R}^{n}$ with apex $o$ which form with $C$ an angle of size at most $\varepsilon$. Clearly, $D_{\varepsilon}(C)$ is a closed cone (not necessarily convex) with apex $o$. For the following lemma, we recall that $S_{L}$ denotes the subspace which is a translate of a plane $L \subset \mathbb{R}^{n}$.

Lemma 3.1. If $L \subset \mathbb{R}^{n}$ is a plane asymptote of a closed convex set $K \subset \mathbb{R}^{n}$, then $\{o\} \neq \operatorname{rec} K \cap S_{L} \not \subset \operatorname{lin} K$. Furthermore, rec $K \cap S_{L} \neq S_{L}$.

Proof. The assertion $\{o\} \neq \operatorname{rec} K \cap S_{L} \not \subset \operatorname{lin} K$ is proved in [9]. Next, assume for a moment that $\operatorname{rec} K \cap S_{L}=S_{L}$. Then $S_{L} \subset \operatorname{rec} K$, and a combination of (P1) and (P4) would imply the inclusion $S_{L} \subset \operatorname{lin} K$, contrary to the first assertion.

We will need one more technical lemma.

Lemma 3.2. Let $K \subset \mathbb{R}^{n}$ be a unbounded closed convex set, and $u \in K$, $v \in \mathbb{R}^{n}$ be points. Any unbounded sequence of points $x_{1}, x_{2}, \ldots \in K \backslash\{u, v\}$ contains a subsequence, say $x_{1}^{\prime}, x_{2}^{\prime}, \ldots$, such that the unit vectors

$$
e_{i}=\frac{x_{i}^{\prime}-u}{\left\|x_{i}^{\prime}-u\right\|} \quad \text { and } \quad c_{i}=\frac{x_{i}^{\prime}-v}{\left\|x_{i}^{\prime}-v\right\|}, \quad i \geq 1,
$$

converge to the same unit vector $e \in \operatorname{rec} K$.

Proof. Since all vectors $\left(x_{i}-u\right) /\left\|x_{i}-u\right\|, i \geq 1$, belong to the unit sphere of $\mathbb{R}^{n}$, a compactness argument implies the existence of a subsequence, say $x_{1}^{\prime}, x_{2}^{\prime}, \ldots$, of $x_{1}, x_{2}, \ldots$ such that the vectors $e_{1}, e_{2}, \ldots$ given by (1) converge to a unit vector $e$. The inequalities

$$
\begin{aligned}
&\left\|x_{i}^{\prime}\right\|-\|u\| \leq\left\|x_{i}^{\prime}-u\right\| \leq\left\|x_{i}^{\prime}\right\|+\|u\|, \\
&\left\|x_{i}^{\prime}\right\|-\|v\| \leq\left\|x_{i}^{\prime}-v\right\| \leq\left\|x_{i}^{\prime}\right\|+\|v\|
\end{aligned}
$$


give

$$
\lim _{i \rightarrow \infty}\left\|x_{i}^{\prime}-u\right\|=\lim _{i \rightarrow \infty}\left\|x_{i}^{\prime}-v\right\|=\infty \quad \text { and } \quad \lim _{i \rightarrow \infty} \frac{\left\|x_{i}^{\prime}-u\right\|}{\left\|x_{i}^{\prime}-v\right\|}=1 .
$$

Therefore,

$$
\begin{aligned}
\lim _{i \rightarrow \infty} c_{i} & =\lim _{i \rightarrow \infty}\left(\frac{x_{i}^{\prime}-u}{\left\|x_{i}^{\prime}-v\right\|}+\frac{u-v}{\left\|x_{i}^{\prime}-v\right\|}\right) \\
& =\lim _{i \rightarrow \infty}\left(\frac{\left\|x_{i}^{\prime}-u\right\|}{\left\|x_{i}^{\prime}-v\right\|} \frac{x_{i}^{\prime}-u}{\left\|x_{i}^{\prime}-u\right\|}+\frac{u-v}{\left\|x_{i}^{\prime}-v\right\|}\right)=1 e+o=e .
\end{aligned}
$$

Next, we assert that the closed halfline $[u, u+e\rangle$ lies in $K$. Indeed, choose any point $y$ in $[u, u+e\rangle$. Then

$$
y=(1-\lambda) u+\lambda(u+e)=u+\lambda e, \quad \lambda \geq 0 .
$$

Clearly, $\|y-u\|=\lambda$. Choose an integer $i_{0}$ such that $\left\|x_{i}^{\prime}-u\right\| \geq \max \{1, \lambda\}$ for all $i \geq i_{0}$. Then

$$
u+e_{i}=\left(1-\frac{1}{\left\|x_{i}^{\prime}-u\right\|}\right) u+\frac{1}{\left\|x_{i}^{\prime}-u\right\|} x_{i}^{\prime} \in\left[u, x_{i}^{\prime}\right], \quad i \geq i_{0} .
$$

With $y_{i}=u+\lambda e_{i}$, one has

$$
y_{i}=(1-\lambda) u+\lambda\left(u+e_{i}\right) \in\left[u, u+e_{i}\right\rangle=\left[u, x_{i}^{\prime}\right\rangle .
$$

Now, the inequality

$$
\left\|y_{i}-u\right\|=\lambda \leq\left\|x_{i}^{\prime}-u\right\|, \quad i \geq i_{0},
$$

gives $y_{i} \in\left[u, x_{i}^{\prime}\right] \subset K$ for all $i \geq i_{0}$. Finally,

$$
y=u+\lambda e=\lim _{i \rightarrow \infty}\left(u+\lambda e_{i}\right)=\lim _{i \rightarrow \infty} y_{i} \in K .
$$

Summing up, $[u, u+e\rangle \subset K$. Finally, (P2) shows that $e \in \operatorname{rec} K$.

Theorem 3.3. Let $K \subset \mathbb{R}^{n}$ be a closed convex set, and let $L \subset \mathbb{R}^{n}$ be a plane asymptote of $K$. With $C=\operatorname{rec} K \cap S_{L}$, the following assertions hold.

(a) For any point $v \in L$ and a scalar $\varepsilon>0$, the closed cone $v+D_{\varepsilon}(C) \cap S_{L}$ lies in $L$ and is an asymptote of $K$. Furthermore, there is a scalar $\varepsilon_{0}>0$ such that $v+D_{\varepsilon}(C) \cap S_{L}$ is a proper subset of $L$ for all $0<\varepsilon<\varepsilon_{0}$. 
(b) If a closed subset $M$ of $L$ is an asymptote of $K$, then, for any point $v \in L$ and a scalar $\varepsilon>0$, the closed set $M \cap\left(v+D_{\varepsilon}(C) \cap S_{L}\right)$ is an asymptote of $K$.

Proof. (a) Since rec $K$ is a closed convex cone with apex $o$, so is the set $C$. By Lemma 3.1. $\{o\} \neq C \neq S_{L}$. Then the polar cone $C_{L}^{\circ}$ of $C$ within the space $S_{L}$ is nontrivial. If $e$ is a nonzero vector in $C_{L}^{\circ}$, then $C$ lies in the closed halfplane $P=\left\{x \in S_{L}: x \cdot e \leq 0\right\}$ of $S_{L}$. A simple geometric argument shows the existence of a scalar $\varepsilon_{0}>0$ such that the closed cone $D_{\varepsilon}(C) \cap S_{L}$ is a proper subset of $S_{L}$ for all $0<\varepsilon<\varepsilon_{0}$.

Obviously, $v+D_{\varepsilon}(C) \cap S_{L}$ is a closed cone with apex $v$. Furthermore,

$$
v+D_{\varepsilon}(C) \cap S_{L} \subset v+S_{L}=L .
$$

By the above argument, $v+D_{\varepsilon}(C) \cap S_{L}$ is a proper subset of $L$ for all $0<\varepsilon<\varepsilon_{0}$.

Because $L$ is an asymptote of $K$, there are sequences of points $p_{1}, p_{2}$, $\cdots \in K$ and $q_{1}, q_{2}, \ldots \in L$ such that $\lim _{i \rightarrow \infty}\left\|p_{i}-q_{i}\right\|=0$. Clearly, both sets $\left\{p_{1}, p_{2}, \ldots\right\}$ and $\left\{q_{1}, q_{2}, \ldots\right\}$ are unbounded. Indeed, otherwise, one could choose respective subsequences converging to the same point in $K \cap L$, contrary to the assumption $K \cap L=\varnothing$.

Choose a point $u \in K$. By Lemma 3.2, there is an unbounded subsequence $p_{i_{1}}, p_{i_{2}}, \ldots$ such that the unit vectors

$$
e_{i_{j}}=\frac{p_{i_{j}}-u}{\left\|p_{i_{j}}-u\right\|} \quad \text { and } \quad c_{i_{j}}=\frac{p_{i_{j}}-v}{\left\|p_{i_{j}}-v\right\|}, \quad j \geq 1,
$$

converge to the same unit vector $e \in \operatorname{rec} K$. Let

$$
c_{i_{j}}^{\prime}=\frac{q_{i_{j}}-v}{\left\|q_{i_{j}}-v\right\|}, \quad j \geq 1 .
$$

Since the subsequence $q_{i_{1}}, q_{i_{2}}, \ldots$ is unbounded and $\left\|p_{i_{j}}-q_{i_{j}}\right\| \rightarrow 0$ as $j \rightarrow \infty$, the inequalities

$$
\left\|q_{i_{j}}-v\right\|-\left\|p_{i_{j}}-q_{i_{j}}\right\| \leq\left\|p_{i_{j}}-v\right\| \leq\left\|q_{i_{j}}-v\right\|+\left\|p_{i_{j}}-q_{i_{j}}\right\|
$$

give

$$
\lim _{j \rightarrow \infty} \frac{\left\|p_{i_{j}}-v\right\|}{\left\|q_{i_{j}}-v\right\|}=1
$$


Therefore,

$$
\begin{aligned}
\lim _{j \rightarrow \infty} c_{i_{j}}^{\prime} & =\lim _{j \rightarrow \infty}\left(\frac{p_{i_{j}}-v}{\left\|q_{i_{j}}-v\right\|}-\frac{p_{i_{j}}-q_{i_{j}}}{\left\|q_{i_{j}}-v\right\|}\right) \\
& =\lim _{j \rightarrow \infty}\left(\frac{\left\|p_{i_{j}}-v\right\|}{\left\|q_{i_{j}}-v\right\|} \frac{p_{i_{j}}-v}{\left\|p_{i_{j}}-v\right\|}-\frac{p_{i_{j}}-q_{i_{j}}}{\left\|q_{i_{j}}-v\right\|}\right)=1 e+o=e .
\end{aligned}
$$

Because $c_{i_{j}}^{\prime} \in S_{L}$ for all $j \geq 1$, the limit vector $e$ also belongs to $S_{L}$. Thus $e \in \operatorname{rec} K \cap S_{L}=C$. Clearly, there is an index $r \geq 1$ such that every vector $e_{i_{j}}, j \geq r$, forms with $e$ an angle of size at most $\varepsilon$. Equivalently, every halfline $\left[o, q_{i_{j}}-v\right\rangle$ forms with the halfline $h=[o, e\rangle \subset C$ an angle of size at most $\varepsilon$. Thus

$$
q_{i_{j}} \in\left[v, q_{i_{j}}\right\rangle=v+\left[o, q_{i_{j}}-v\right\rangle \subset v+D_{\varepsilon}(h) \subset v+D_{\varepsilon}(C) \cap S_{L}, \quad j \geq r .
$$

Finally, because $\lim _{j \rightarrow \infty}\left\|p_{i_{j}}-q_{i_{j}}\right\|=0$, the cone $v+D_{\varepsilon}(C) \cap S_{L}$ is an asymptote of $K$.

(b) Suppose that a closed subset $M$ of $L$ is an asymptote of $K$. Then there are sequences of points $p_{1}, p_{2}, \ldots \in K$ and $q_{1}, q_{2}, \ldots \in M$ such that $\lim _{i \rightarrow \infty}\left\|p_{i}-q_{i}\right\|=0$. An obvious modification of the argument from part (a) shows the existence of unbounded subsequences

$$
p_{i_{1}}, p_{i_{2}}, \ldots \in K \quad \text { and } \quad q_{i_{1}}, q_{i_{2}}, \ldots \in M \cap\left(v+D_{\varepsilon}(C) \cap S_{L}\right)
$$

such that $\lim _{j \rightarrow \infty}\left\|p_{i_{j}}-q_{i_{j}}\right\|=0$. So, the set $M \cap\left(v+D_{\varepsilon}(C) \cap S_{L}\right)$ is an asymptote of $K$.

We observe that, generally, Theorem 2.1 does not follow from Theorem 3.3. Indeed, if $K$ is the convex set from Example 2.2, then its recession cone is the polyhedron $\{(x, y, z): x \geq 0, y \geq 0\}$. In terms of Theorem 3.3 . $S_{L}=L, C$ is the halfplane $\{(x, 0, z): x \geq 0\}$ of $L$, and $v+D_{\varepsilon}(C) \cap S_{L}$ is the nonconvex closed cone in $L$ of angle size $\pi+2 \varepsilon$. Clearly, this cone is larger than any halfplane asymptote of $K$ contained in $v+D_{\varepsilon}(C) \cap S_{L}$, and there is no halfplane asymptote of $K$ containing this cone.

Analysis of the proof of Theorem 3.3 results in the following corollary, which shows the existence (but not a constructive description) of arbitrarily sharp pencil cone asymptotes.

Corollary 3.4. Let $K \subset \mathbb{R}^{n}$ be a closed convex set, and $L \subset \mathbb{R}^{n}$ be a plane asymptote of $K$. There is a closed halfline $h \subset \operatorname{rec} K \cap S_{L}$ with endpoint $o$ such that for any point $v \in L$ and scalar $\varepsilon>0$, the pencil cone $\left(v+D_{\varepsilon}(h)\right) \cap L$ is an asymptote of $K$. 
The next example shows that in Theorem 3.3 , the set $D_{\varepsilon}(C)$ cannot be replaced with $C$ and even with the closed metric $\varepsilon$-neighborhood of $C$, defined by

$$
N_{\varepsilon}(C)=\left\{x \in \mathbb{R}^{n}: \rho(x, C) \leq \varepsilon\right\} .
$$

Consequently, no translate of the cone $v+C$ may be an asymptote of $K$.

Example 3.5 . The set $K \subset \mathbb{R}^{3}$ given by the conditions

$$
K=\left\{(x, y, z): x>0, y \geq 1 / x, z \geq(x+y)^{2}\right\}
$$

is closed and convex. Furthermore, the following properties of $K$ hold:

(a) the coordinate $x z$-plane, say $L$, is an asymptote of $K$;

(b) no closed slab between a pair of parallel lines in $L$ is an asymptote of $K$;

(c) $\operatorname{rec} K$ is the vertical halfline $h=\{(0,0, z): z \geq 0\}$, and no metric neighborhood $v+N_{\varepsilon}(h) \cap L$ of the halfline $v+h$, with $v \in L$, is an asymptote of $K$;

(d) for any choice of scalars $\alpha \geq 0$, and $\beta, \gamma \in \mathbb{R}$, the planar cone

$$
D(\alpha, \beta, \gamma)=\{(x, 0, z): z \geq \alpha|x-\beta|+\gamma\} \subset L
$$

is an asymptote of $K$.

Indeed, the set $K$ is closed and convex as the intersection of closed and convex sets

$$
K_{1}=\{(x, y, z): x>0, y \geq 1 / x\} \quad \text { and } \quad K_{2}=\left\{(x, y, z): z \geq(x+y)^{2}\right\} .
$$

(a) Clearly, $K \cap L=\varnothing$, and the sequences of points

$$
p_{i}=\left(i, 1 / i,\left[\left(i^{2}+1\right) / i\right]^{2}\right) \in K, \quad q_{i}=\left(i, 0,\left[\left(i^{2}+1\right) / i\right]^{2}\right) \in L, \quad i \geq 1,
$$

satisfy the condition $\left\|p_{i}-q_{i}\right\|=1 / i$. So, $\delta(K, L)=0$, which shows that $L$ is an asymptote of $K$.

(b) Let $Q$ be any closed slab between a pair of parallel lines in $L$.

Assume first that $Q$ is vertical. Then it can be described as

$$
Q=\{(x, 0, z): \alpha \leq x \leq \beta\}, \quad \text { where } \alpha \leq \beta
$$


Since $K_{1} \cap Q=\varnothing$ and $K_{1}$ is a both-way unbounded vertical cylinder, $\delta\left(K_{1}, Q\right)$ equals the distance between the branch of hyperbola $\{(x, y, 0): x>0, x y=1\}$ $\subset$ bd $K_{1}$ and the point $(\alpha, 0,0) \in Q$, which is positive. Therefore, the inclusion $K \subset K_{1}$ gives $\delta(K, Q) \geq \delta\left(K_{1}, Q\right)>0$. Hence no vertical slab in $L$ is an asymptote of $K$.

Suppose now that $Q$ is slant. Then

$$
Q=\left\{(x, 0, z): \alpha x+\beta \leq z \leq \alpha x+\beta^{\prime}\right\}
$$

for suitable scalars $\alpha$ and $\beta \leq \beta^{\prime}$. Assume for a moment that $\delta(K, Q)=0$. Then one can choose sequences of points $p_{i} \in K$ and $q_{i} \in Q, i \geq 1$, such that $\left\|p_{i}-q_{i}\right\| \rightarrow 0$ as $i \rightarrow \infty$. Denote by $p_{i}^{\prime}$ the point at which the segment $\left[p_{i}, q_{i}\right]$ meets bd $K, i \geq 1$. Let

$$
p_{i}^{\prime}=\left(x_{i}, y_{i}, z_{i}\right) \quad \text { and } \quad q_{i}=\left(u_{i}, 0, v_{i}\right), \quad \alpha u_{i}+\beta \leq v_{i} \leq \alpha u_{i}+\beta^{\prime}, \quad i \geq 1 .
$$

By a convexity argument, $x_{i} y_{i}=1$ and $\left\|p_{i}^{\prime}-q_{i}\right\| \leq\left\|p_{i}-q_{i}\right\|$. Hence $\left\|p_{i}^{\prime}-q_{i}\right\| \rightarrow$ 0 as $i \rightarrow \infty$, or, equivalently,

$$
\lim _{i \rightarrow \infty}\left(\left(x_{i}-u_{i}\right)^{2}+1 / x_{i}^{2}+\left(z_{i}-v_{i}\right)^{2}\right)=0 .
$$

Thus $x_{i} \rightarrow \infty$ and $u_{i}=x_{i}+\varepsilon_{i}$, where $\varepsilon_{i} \rightarrow 0$ as $i \rightarrow \infty$. Consequently, there is an index $i_{0} \geq 0$ and a scalar $\gamma>0$ such that

$$
\begin{aligned}
\left|v_{i}\right| & \leq \max \left\{\left|\alpha u_{i}+\beta\right|,\left|\alpha u_{i}+\beta^{\prime}\right|\right\} \\
& \leq\left|\alpha x_{i}\right|+\left|\alpha \varepsilon_{i}\right|+\max \left\{|\beta|,\left|\beta^{\prime}\right|\right\} \leq\left|\alpha x_{i}\right|+\gamma
\end{aligned}
$$

whenever $i \geq i_{0}$. Therefore,

$$
\left|z_{i}-v_{i}\right| \geq\left|z_{i}\right|-\left|v_{i}\right| \geq\left(\left(x_{i}+1 / x_{i}\right)^{2}-\left|\alpha x_{i}\right|-\gamma\right) \rightarrow \infty \quad \text { as } i \rightarrow \infty,
$$

in contradiction with (3). Summing up, $Q$ cannot be an asymptote of $K$.

(c) Given the point $p=(1,1,4) \in K$, the halfline $p+h=\{(1,1, z): z \geq 4\}$ is included in $K$. This argument and (P2) imply that $h \subset \operatorname{rec} K$.

For the opposite inclusion, choose any vector $e=(u, v, w) \in \operatorname{rec} K$. Then

$$
p+\lambda e=(1+\lambda u, 1+\lambda v, 4+\lambda w) \in K \quad \text { for all } \lambda>0 .
$$

Equivalently, for all $\lambda>0$, one has

$$
1+\lambda u>0, \quad 1+\lambda v \geq 1 /(1+\lambda u), \quad 4+\lambda w \geq(2+\lambda(u+v))^{2} .
$$


The first condition implies that $u \geq 0$. This inequality and the second condition give $v \geq 0$, while the third condition results in $u+v=0$. Hence $u=v=0$ and $w \geq 0$, implying the inclusion $e \in h$. Summing up, $\operatorname{rec} K=h$.

It is easy to see that a metric neighborhood $v+N_{\varepsilon}(h) \cap L$ of $v+h$, with $v \in L$, is a subset of a suitable vertical slab $Q \subset L$. According to part (b) above, $Q$ is not an asymptote of $K$. Hence $v+N_{\varepsilon}(h) \cap L$ is not an asymptote of $K$.

(d) Let $S_{L}=L$ and $v=(\beta, 0, \gamma)$. If $\alpha>0$, then the planar cone $D(\alpha, \beta, \gamma)$ from (2) can be expressed as $v+D_{\varepsilon}(h) \cap S_{L}$, where $\varepsilon=\cot ^{-1}(\alpha)$. If $\alpha=0$, then $D(0, \beta, \gamma)=v+D_{\pi / 2}(h) \cap S_{L}$. In either case, Corollary 3.4 shows that $D(\alpha, \beta, \gamma)$ is an asymptote of $K$.

\section{Line-FREE CONE ASYMPtoteS}

We describe below a family of plane asymptotes $M$ contained in a plane asymptote $L$ of $K$ such that the cone $D_{\varepsilon}(\operatorname{rec} K \cap M) \cap S_{M}$ becomes line-free provided $\varepsilon$ is sufficiently small.

Theorem 4.1. Let $K \subset \mathbb{R}^{n}$ be a closed convex set, $L \subset \mathbb{R}^{n}$ be a plane asymptote of $K$, and $F$ be a subspace complementary to $\operatorname{lin} K \cap S_{L}$ in $S_{L}$. The following assertions hold.

(a) For any point $v \in L$, the plane $M=v+F$ lies in $L$ and is an asymptote of $K$.

(b) For any point $v \in L$ and any $\varepsilon>0$, the closed cone $v+D_{\varepsilon}(\operatorname{rec} K \cap F) \cap F$ is a line-free asymptote of $K$.

(c) There is an n-dimensional line-free closed convex cone $C^{\prime} \subset \mathbb{R}^{n}$ with apex o such that $C^{\prime} \subset D_{\varepsilon}(\operatorname{rec} K \cap F)$ and the cone $v+C^{\prime} \cap F$ is an asymptote of $K$.

The proof of Theorem 4.1 is divided into Lemmas $4.2-4.4$.

Lemma 4.2. Let $K \subset \mathbb{R}^{n}$ be a closed convex set, $L \subset \mathbb{R}^{n}$ be a plane asymptote of $K$, and $v$ be a point in $L$. For any subspace $F \subset \mathbb{R}^{n}$ complementary to $\operatorname{lin} K \cap S_{L}$ in $S_{L}$, the plane $M=v+F$ lies in $L$ and is an asymptote of $K$.

Proof. Since $\operatorname{lin} K \subset \operatorname{rec} K$, Lemma 3.1 shows that the subspace $T=$ $\operatorname{lin} K \cap S_{L}$ is distinct from $S_{L}$ (possibly, $T=\{o\}$ ). By (P3), $K=K+T$. 
Choose a subspace $F \subset \mathbb{R}^{n}$ complementary to $T$ in $S_{L}$ and consider the plane $M=v+F$. Since $M=v+F \subset v+S_{L}=L$ and $K \cap L=\varnothing$, it remains to show that $\delta(K, M)=0$. Indeed, choose sequences of points $p_{1}, p_{2}, \ldots \in K$ and $q_{1}, q_{2}, \ldots \in L$ such that $\lim _{i \rightarrow \infty}\left\|p_{i}-q_{i}\right\|=0$. Clearly, $q_{i}-v \in S_{L}$, $i \geq 1$. Denote by $q_{i}^{\prime}$ the projection of $q_{i}-v$ on $F$ in the direction of $T$, and let $p_{i}^{\prime}=p_{i}+\left(v+q_{i}^{\prime}-q_{i}\right), i \geq 1$. Because

$$
v+q_{i}^{\prime}-q_{i}=q_{i}^{\prime}-\left(q_{i}-v\right) \in T,
$$

one has

$$
p_{i}^{\prime}=p_{i}+\left(v+q_{i}^{\prime}-q_{i}\right) \in K+T=K, \quad i \geq 1 .
$$

Furthermore, $v+q_{i}^{\prime} \in v+F=M, i \geq 1$, and

$$
\begin{aligned}
\delta(K, M) & \leq \lim _{i \rightarrow \infty}\left\|p_{i}^{\prime}-\left(v+q_{i}^{\prime}\right)\right\| \\
& =\lim _{i \rightarrow \infty}\left\|\left(p_{i}+v+q_{i}^{\prime}-q_{i}\right)-\left(v+q_{i}^{\prime}\right)\right\| \\
& =\lim _{i \rightarrow \infty}\left\|p_{i}-q_{i}\right\|=0 .
\end{aligned}
$$

Summing up, $M$ is an asymptote of $K$.

Lemma 4.3. Let $K \subset \mathbb{R}^{n}$ be a closed convex set, and $L \subset \mathbb{R}^{n}$ be a plane asymptote of $K$. Given a subspace $F$ complementary to $\operatorname{lin} K \cap S_{L}$ in $S_{L}$, the closed convex cone $C=\operatorname{rec} K \cap F$ with apex $o$ is nontrivial and line-free.

Proof. By Lemma 4.2, every translate of the form $v+F$, where $v \in L$, is an asymptote of $K$. Consequently, Lemma 3.1 shows that the closed convex cone $C=\operatorname{rec} K \cap F$ is distinct from $\{o\}$. It remains to prove that $C$ is line-free. Assume for a moment that $C$ contains a line $l$. We consider separately the following two cases.

(i) Let $o \in l$. By (P4), $l$ is a 1-dimensional subspace contained in $\operatorname{lin} K$. Consequently, $l \subset \operatorname{lin} K \cap F$, contrary to the choice of $F$.

(ii) Let $o \notin l$. Because $o$ is an apex of $\operatorname{rec} K$, every closed halfline $[o, x\rangle$, where $x \in l$, is contained in $\operatorname{rec} K$. Clearly, the union of all such halflines coincides with $\{o\} \cup P$, where $P$ is the open halfplane of the 2-dimensional subspace, $\operatorname{span} l$, bounded by the 1 -dimensional subspace $l^{\prime}=l-l$. Then $l^{\prime} \subset \operatorname{cl} P \subset \operatorname{rec} K$, and, as above, $l^{\prime} \in \operatorname{lin} K \cap F$, contrary to the choice of $F$.

Summing up, $C$ should be line-free. 
Lemma 4.4. If $C \subset \mathbb{R}^{n}$ is a closed line-free convex cone with apex o, then there is a scalar $\varepsilon>0$ such that the closed cone $D_{\varepsilon}(C)$ is line-free. Furthermore, there is an n-dimensional closed convex cone $C^{\prime} \subset \mathbb{R}^{n}$ with apex $o$ and a scalar $\varepsilon^{\prime} \in(0, \varepsilon)$ such that $D_{\varepsilon^{\prime}}(C) \subset C^{\prime} \subset D_{\varepsilon}(C)$.

Proof. Since $C$ is line-free, the polar cone $C^{\circ}$ is $n$-dimensional (see, e.g., [10, Theorem 8.4]). Choose a nonzero vector $e \in\left(-\operatorname{int} C^{\circ}\right)$. Then $C \backslash\{o\}$ is contained in the open halfspace $W=\left\{x \in \mathbb{R}^{n}: x \cdot e>0\right\}$ (see [10, Theorem 8.6]). Denote by $\mathbb{S}$ the unit sphere of $\mathbb{R}^{n}$, and let $E=C \cap \mathbb{S}$. Clearly, $C=\{\lambda x: \lambda \geq 0, x \in E\}$.

Since $E$ is a compact subset of $W$, there is an $\varepsilon>0$ such that the closed set

$$
V_{\rho}(E)=\{x \in \mathbb{S}: \delta(x, E) \leq \rho\}, \quad \rho=2 \sin (\varepsilon / 2),
$$

is contained in $W$. We observe that a closed halfline $h=[o, u\rangle$, with $u \in \mathbb{S}$, is contained in $D_{\varepsilon}(C)$ if and only if there is a closed halfline $h^{\prime}=\left[o, u^{\prime}\right\rangle$, with $u^{\prime} \in E$, such that the angle between $h$ and $h^{\prime}$ is of size at most $\varepsilon$. Considering the isosceles triangle $\Delta\left(o, u, u^{\prime}\right)$, we deduce that the angle between $h$ and $h^{\prime}$ is of size at most $\varepsilon$ if and only if $\left\|u-u^{\prime}\right\| \leq 2 \sin (\varepsilon / 2)$. This argument implies that

$$
D_{\varepsilon}(C)=\left\{\lambda x: \lambda \geq 0, x \in V_{\rho}(E)\right\} .
$$

It is easy to see that every closed halfline $[o, x\rangle$, where $x \in V_{\rho}(E)$, is contained in $\{o\} \cup W$. Hence $D_{\varepsilon}(C) \subset\{o\} \cup W$. An argument similar to that of Lemma 4.3 shows that the cone $D_{\varepsilon}(C)$ is line-free.

Finally, consider the hyperplane $H=\left\{x \in \mathbb{R}^{n}: x \cdot e=1\right\}$. Then both sets $A=C \cap H$ and $B=D_{\varepsilon}(C) \cap H$ are compact (see [10, Theorem 8.15]), and $A$ is convex. Furthermore, both sets $A$ and $B$ have dimension $n-1$ and $\operatorname{rint} A \subset \operatorname{rint} B$ (see [10, Theorem 8.14]). Given any point $a \in \operatorname{rint} A$, the set $A^{\prime}=a+\mu(A-a)$, with $\mu>1$, is convex and contains $A$ in its relative interior. A compactness argument implies that $A^{\prime} \subset B$ provided $\mu$ is sufficiently close to 1 . For this value of $\mu$, let

$$
C^{\prime}=C_{o}\left(A^{\prime}\right)=\left\{\lambda x: \lambda \geq 0, x \in A^{\prime}\right\} .
$$

Then $C^{\prime}$ is a closed convex cone satisfying the inclusions $C \subset\{o\} \cup \operatorname{int} C^{\prime}$ and $C^{\prime} \subset D_{\varepsilon}(C)$, as desired.

The following example shows that the cone $D_{\varepsilon}(C)$ in Lemma 4.4 may be nonconvex even if the cone $C$ is convex and line-free. 
Example 4.5. Let $C=\{(x, y, 0): y \geq|x| / 10\}$ be the planar cone in $\mathbb{R}^{3}$. Clearly, $C$ is closed, convex, and line-free. Let $\varepsilon=\sin ^{-1}(0.1) \approx 0.1$. The point $u=(10,1,1)$ belongs to $D_{\varepsilon}(C)$ because $u^{\prime}=(10,1,0) \in C$ and the angle between the halflines $[o, u\rangle$ and $\left[o, u^{\prime}\right\rangle$ equals $\sin ^{-1}(1 / \sqrt{102})<\varepsilon$. Similarly, the point $v=(-10,1,1)$ belongs to $D_{\varepsilon}(C)$. On the other hand, the midpoint $w=(0,1,1)$ of the segment $[u, v]$ does not belong to $D_{\varepsilon}(C)$. Indeed, the angle between $[o, w\rangle$ and $C$ is achieved on the pair of halflines $[o, w\rangle$ and $\left[o, w^{\prime}\right\rangle$, where $w=(0,1,0)$, and this angle equals $\sin ^{-1}(1 / \sqrt{2})=\pi / 4>\varepsilon$. So, $w \notin D_{\varepsilon}(C)$, implying that the cone $D_{\varepsilon}(C)$ is not convex.

In view of Theorem 4.1, one may ask about the existence of minimal (under inclusion) cone asymptotes of $K$. The following theorem shows that such asymptotes (if any) should be halflines.

Theorem 4.6. Let $X \subset \mathbb{R}^{n}$ be a nonempty closed set, and $C \subset \mathbb{R}^{n}$ be a closed convex cone with apex $v$, which is an asymptote of $X$. If $\operatorname{dim} C \geq 2$, then there is a convex cone $C^{\prime}$ with apex $v$ which is an asymptote of $K$ and a proper subset of $C$.

Proof. Choose a point $u \in \operatorname{rint} C \backslash\{v\}$, and consider a hyperplane $H \subset \mathbb{R}^{n}$ containing $\{u, v\}$ such that $C$ meets the interiors of both closed halfspaces, say $V_{1}$ and $V_{2}$, determined by $H$. The sets $C_{1}=C \cap V_{1}$ and $C_{2}=C \cap V_{2}$ are closed convex cones with common apex $v$, whose union is $C$, and each of these cones is a proper subset of $C$. Because $C$ is an asymptote of $K$, there is an unbounded sequence of points $x_{1}, x_{2}, \ldots \in C$ such that $\delta\left(x_{i}, X\right) \rightarrow 0$ as $i \rightarrow \infty$. Clearly, one of the cones $C_{1}$ and $C_{2}$, say $C_{1}$, contains an infinite subsequence $x_{1}^{\prime}, x_{2}^{\prime}, \ldots$ of $x_{1}, x_{2}, \ldots$. Since $C_{1} \cap X \subset C \cap X=\varnothing$ and $\delta\left(x_{i}^{\prime}, X\right) \rightarrow 0$ as $i \rightarrow \infty$, the cone $C_{1}$ is an asymptote of $X$.

\section{Properties of CONe Asymptotes}

The next two theorems show that some properties of plane asymptotes can be generalized to the case of cone asymptotes (see [4] and [9] for the original statements).

Theorem 5.1. Let $C \subset \mathbb{R}^{n}$ be a closed convex cone with apex o. If a translate of $C$ is an asymptote of a closed convex set $K \subset \mathbb{R}^{n}$, then $\operatorname{rec} K \cap C \neq\{o\}$. Furthermore, for any nonzero vector $e \in \operatorname{rec} K \cap C$, there is a translate of the closed halfline $h=[o, e\rangle$ which either lies in bd $K$ or is an asymptote of $K$. 
Proof. Let a translate $D=v+C$ of $C$ be an asymptote of $K$. Then $K \cap D=\varnothing$ and there are sequences of points $p_{1}, p_{2}, \ldots \in K$ and $q_{1}, q_{2}, \ldots \in D$ such that $\lim _{i \rightarrow \infty}\left\|p_{i}-q_{i}\right\|=0$. We observe that both sequences are unbounded. Indeed, otherwise one could choose respective subsequences converging to the same point in $K \cap D$, contrary to the assumption $K \cap D=\varnothing$. As shown in the proof of Theorem 3.3 , there are subsequences $p_{i_{1}}, p_{i_{2}}, \ldots$ and $q_{i_{1}}, q_{i_{2}}, \ldots$ such that the unit vectors

$$
e_{i_{j}}=\frac{p_{i_{j}}-u}{\left\|p_{i_{j}}-u\right\|} \quad \text { and } \quad c_{i_{j}}^{\prime}=\frac{q_{i_{j}}-v}{\left\|q_{i_{j}}-v\right\|}, \quad j \geq 1,
$$

converge to the same unit vector $e \in \operatorname{rec} K$. Since $q_{i_{j}}-v \in C$ for all $j \geq 1$ and the cone $C$ is closed, we obtain that $e \in C$.

Consider the halfline $h=[o, e\rangle$. Then $h \subset C$ and, by (P2), the halfline $u+h$ lies in $K$. Consider the family of parallel halflines

$$
h(\lambda)=((1-\lambda) v+\lambda u)+h, \quad 0 \leq \lambda \leq 1 .
$$

By the above, $h(0) \subset D$ and $h(1) \subset K$. Consequently, $K \cap h(0)=\varnothing$. Let

$$
\lambda^{\prime}=\sup \{\lambda \in[0,1]: K \cap h(\lambda)=\varnothing\} .
$$

It is easy to see that int $K \cap h\left(\lambda^{\prime}\right)=\varnothing$ and $\delta\left(K, h\left(\lambda^{\prime}\right)\right)=0$. If $K \cap h\left(\lambda^{\prime}\right)=\varnothing$, then $h\left(\lambda^{\prime}\right)$ is an asymptote of $K$. Suppose that $K \cap h\left(\lambda^{\prime}\right) \neq \varnothing$ and choose a point $w \in K \cap h(\lambda)$. By (P2), the halfline $w+h$ lies in $K$, and the inclusion $w+h \subset h\left(\lambda^{\prime}\right)$ implies that $w+h \subset$ bd $K$, as desired.

The following example shows that in Theorem 5.1 (unlike Lemma 3.1) the inclusion $\operatorname{rec} K \cap C \subset \operatorname{lin} K$ is possible.

EXAmPle 5.2. Let the closed convex sets $K \subset \mathbb{R}^{3}$ be given by

$$
K=\{(x, y, z): x \geq 0, y \geq 0, x+y \geq 1\} .
$$

Clearly, $\operatorname{rec} K=\{(x, y, z): x \geq 0, y \geq 0\}$ and $\operatorname{lin} K$ is the $z$-axis. Let $C$ be the closed convex cone with apex $o$ generated by the circular disk

$$
D=\left\{(x, y, 1): x^{2}+(y+1)^{2} \leq 1\right\} .
$$

The cone $C$ is an asymptote of $K$ because $K \cap C=\varnothing$ and $\delta(C, l)=0$, where $l \subset K$ is the vertical line $\{(1,0, z): z \in \mathbb{R}\}$. At the same time, $\operatorname{rec} K \cap C=\{(0,0, z): z \geq 0\}$ is a subset of $\operatorname{lin} K$. 
TheOREM 5.3. Let $X \subset \mathbb{R}^{n}$ be a nonempty closed set and $C \subset \mathbb{R}^{n}$ be a closed convex cone with apex $o$. There is a translate of $C$ which is an asymptote of $X$ if and only if the set $X-C$ is not closed.

Proof. Let a translate $D=v+C$ of $C$ be an asymptote of $X$. We first observe that $v \notin X-C$. Indeed, assume for a moment that $v \in X-C$. Then $v=x-u$, where $x \in X$ and $u \in C$. Consequently, $x=v+u \in v+C=D$, contrary to the assumption $D \cap X=\varnothing$. Since $D$ is an asymptote of $X$, there are sequences of points $x_{1}, x_{2}, \ldots \in X$ and $y_{1}, y_{2}, \ldots \in D$ such that $\lim _{i \rightarrow \infty}\left\|x_{i}-y_{i}\right\|=0$. Expressing $y_{i}$ as $y_{i}=v+z_{i}$ for a suitable $z_{i} \in C, i \geq 1$, one has $x_{i}-z_{i} \in X-C$ and

$$
\lim _{i \rightarrow \infty}\left\|v-\left(x_{i}-z_{i}\right)\right\|=\lim _{i \rightarrow \infty}\left\|y_{i}-x_{i}\right\|=0
$$

So, $v \in \operatorname{cl}(X-C) \backslash(X-C)$. Thus the set $X-C$ is not closed.

Conversely, suppose that the set $X-C$ is not closed. Choose a point

$$
v \in \operatorname{cl}(X-C) \backslash(X-C)
$$

and consider the cone $D=v+C$. We assert that $D \cap X=\varnothing$. Indeed, assume the existence of a point $z \in D \cap X$. Then $z=v+u=x$, where $u \in C$ and $x \in X$. Consequently,

$$
v=x-u \in X-C,
$$

which is impossible by the choice of $v$.

Next, the inclusion $v \in \operatorname{cl}(X-C)$ implies the existence of a sequence $u_{1}, u_{2}, \ldots$ of points in $X-C$ converging to $v$. With $u_{i}=x_{i}-z_{i}$, where $x_{i} \in X$ and $z_{i} \in C$, and with $w_{i}=v+z_{i} \in D, i \geq 1$, we obtain that

$$
\lim _{i \rightarrow \infty}\left\|x_{i}-w_{i}\right\|=\lim _{i \rightarrow \infty}\left\|\left(u_{i}+z_{i}\right)-\left(v+z_{i}\right)\right\|=\lim _{i \rightarrow \infty}\left\|u_{i}-v\right\|=0 .
$$

Hence $\delta(X, D)=0$. Summing up, $D$ is an asymptote of $X$.

\section{ACKNOWLEDGEMENTS}

The author is thankful to the referee for the careful reading and considered suggestions leading to a better presented paper. 


\section{REFERENCES}

[1] A. Auslender, M. Teboulle, "Asymptotic Cones and Functions in Optimization and Variational Inequalities", Springer-Verlag, New York, 2003.

[2] D. Gale, V. Klee, Continuous convex sets, Math. Scand. 7 (1959), $379-391$.

[3] P. Goossens, Hyperbolic sets and asymptotes, J. Math. Anal. Appl. 116 (1986), 604-618.

[4] V. KLEE, Asymptotes and projections of convex sets, Math. Scand. 8 (1960), $356-362$.

[5] V.L. KleE, Asymptotes of convex bodies, Math. Scand. 20 (1967), 89-90.

[6] J. Lawrence, V. Soltan, On unions and intersections of nested families of cones, Beitr. Algebra Geom. 57 (2016), 655-665.

[7] J.E. Martínez-Legaz, D. Noll, W. Sosa, Minimization of quadratic functions on convex sets without asymptotes, J. Convex Anal. 25 (2018), $623-641$.

[8] J.E. Martínez-Legaz, D. Noll, W. Sosa, Non-polyhedral extensions of the Frank and Wolfe theorem, in "Splitting Algorithms, Modern Operator Theory, and Applications" (H. Bauschke, R. Burachik, D. Luke editors), Springer, Cham, 2019, 309-329.

[9] V. Soltan, Asymptotic planes and closedness conditions for linear images and vector sums of sets, J. Convex Anal. 25 (2018), 1183-1196.

[10] V. Soltan, "Lectures on Convex Sets", Second edition, World Scientific, Hackensack, NJ, 2020.

[11] V. Soltan, On M-decomposable sets, J. Math. Anal. Appl. 485 (2020), $123816,15 \mathrm{pp}$. 